\title{
Posición de la Estelas 10 y 12 respecto del Parque Arqueológico de Copán Ruinas
}

Roberto Schöngarth, Deborah Forrest,

Edward Milla, Ricardo Pastrana

\section{Resumen}

El equipo encabezado por el antropólogo y arqueólogo Vito Véliz hizo varias observaciones en Copán Ruinas desde el 11 hasta el 14 de agosto de 2010, aprovechando el paso del Sol por el cenit.

Las visitas al Parque Arqueológico consistieron en observar tanto el paso del Sol por el cenit como además tratar de establecer una relación de la posición de las Estelas 10 y 12, fuera del parque, en las montañas cercanas. Las investigaciones en las últimas décadas muestran que hay un trasfondo astronómico en la colocación de la mayoría de las estelas mayas. En el caso de las estelas 10 y 12 se obtuvo las coordenadas geográficas de ambos lugares y se hizo una proyección de la esfera celeste, tal y como pudiesen haberla observado los mayas, tomando en cuenta la precesión y la variación de la inclinación de eje terrestre. La evidencia recopilada muestra que las estelas 10 y 12, fuera del Parque Arqueológico de Copán, no tienen una relación en su posición relativa respecto a algún evento astronómico más que para marcar cada una, independientemente, los límites norte en el caso de la estela 10 y sur en el caso de la estela 12, del Parque Arqueológico de Copán.

Palabras clave: Parque Arqueológico, Copán Ruinas, alineamiento, estelas mayas, trigonometría esférica

Roberto Schöngarth'1, Deborah Forrest' ${ }^{1}$, Edward Milla', Ricardo Pastrana ${ }^{1}$

${ }^{1}$ Maestría Académica Regional Centroamericana en Astronomía y Astrofísica FACES Universidad Nacional Autónoma de Honduras 


\section{Introducción}

El trabajo consiste en los resultados obtenidos con motivo de la visita al Parque Arqueológico de Copán por parte del grupo de estudiantes de la primera promoción de la Maestría Regional Centroamericana en Astronomía y Astrofísica en la asignatura "Seminario de Arqueoastronomía", impartida por el antropólogo y arqueólogo Santos Vito Véliz. El grupo de estudiantes estuvo conformado por Deborah Forrest, Edward Milla, Ricardo Pastrana y Roberto Schöngarth.

El objetivo fundamental consistió en recopilar información acerca de la posición de las estelas 10 y 12, a fin de determinar su posición relativa en relación a los astros.

Para ello, mediante GPS, se tomaron las coordenadas geográficas en ambos lugares para posteriormente elaborar un estudio acerca de la posición de los astros, el Sol y las estrellas en particular, en relación a ambas estelas.

El trabajo aquí presentado evidencia el procedimiento seguido para obtener conclusiones al respecto, tomando en cuenta los movimientos de la Tierra, incluidos los seculares, a fin de tratar de reproducir fielmente el cielo que observaron los mayas hace alrededor de 1500 años.

Si bien éste era el objetivo primordial, la visita de los estudiantes fue extensiva para observar tanto el paso del Sol por el cenit de Copán (Imagen 1), así como en labor divulgativa. Ésta consistió tanto en charlas a maestros de la Escuela Mayatán acerca de astronomía en general, así como una breve conferencia acerca del paso del Sol por el cenit, impartida por Edward Milla en el Hotel Marina de Copán. De igual manera se hizo un sondeo de las creencias populares relativas a los astros con una entrevista en la comunidad de Hacienda Grande, colindante con Copán Ruinas.

\section{Marco teórico}

Los cuerpos celestes han tenido una enorme influencia en el ser humano. Desde épocas antiguas, las distintas culturas encontradas por todo el globo terráqueo presentan evidencias de la utilización de observaciones astronómicas en la agricultura, construcción o religión, involucrando a varias de las culturas más importantes de los últimos milenios como los babilonios, los egipcios, los griegos y las culturas mesoamericanas. 
Los mayas no fueron la excepción. Hoy en día todavía siguen maravillando con su enorme conocimiento acerca de muchos aspectos del ser humano. Al igual que otras civilizaciones, los mayas también fueron grandes observadores del cielo. Hoy se sabe que usaban el movimiento del Sol para orientar sus construcciones y, hace pocos años, se dio a conocer al mundo el descubrimiento del reloj solar (Pineda de Carías, Véliz y Agurcia, 2002).

La civilización Maya ocupó y floreció en un territorio que va desde el sureste de México, extendiéndose hacia el este de Guatemala, El Salvador y el occidente de Honduras. Su territorio se estima entre los $325,000 \mathrm{~km} 2$ a los $400,000 \mathrm{~km} 2$. En este territorio hubo muchas ciudades que alcanzaron un desarrollo excepcional tales como Tikal, Bonampak, Copán, Quiriguá, Palenque, Calakmul, Yaxchilán, Piedras Negras, Altar de Sacrificios y Uaxactún (Sharer 2006: P153-497).

En el sureste del territorio Maya (Occidente de Honduras) y contiguo al Río Copán se ubica una de las ciudades más importantes para antropólogos, arqueólogos, astrónomos y turistas. Es la Ciudad de Copán, declarada por la UNESCO patrimonio de la humanidad. Su ubicación estratégica permitía a los antiguos mayas obtener el sustento gracias a la pesca, caza y agricultura, siendo su principal producto el maíz.

Las investigaciones sobre los mayas han sido y siguen siendo de gran importancia para la humanidad. Las incógnitas que rodean a esta gran civilización aún son muchas, algunas quizá sin repuestas y otras se encuentran en vía de ser contestadas.

\section{Metodología}

El proceso de observación y recolección de datos no implicó manipulación de variables. El estudio consistió en la toma de mediciones asociadas a los puntos donde las hipótesis muestran que pudiese haber una alineación, para posteriormente confirmar la precisión de estas alineaciones.

La toma de datos consistió en determinar las coordenadas geográficas, a través de un GPS, de las posiciones de las estelas 10 y 12. La visita a la estela 10 se realizó durante la mañana del 12 de agosto de 2011 y a la estela 12 la mañana del 13 de agosto, donde además se tomaron fotografías. 
Ya con los datos tomados posteriormente se determinan las distancias y direcciones de una estela respecto a la otra utilizando trigonometría esférica. Este proceso permite además relacionar las coordenadas altacimutales y las coordenadas ecuatoriales y así determinar las posiciones principalmente del Sol, pero además de las otras estrellas, según fuese la época del año en un tiempo asociado a la época cuando la cultura maya poblaba el lugar.

\section{Observaciones en las estelas 10 y 12}

La Imagen 2 muestra la localización de las Estelas 10 y 12 respecto a la comunidad de Copán Ruinas, casi al centro de la imagen, y al Parque Arqueológico del cual aparecen marcadas tanto la Estela D como la Estructura 4. Obsérvese que el Norte está orientado hacia arriba de la imagen.

La visita a la Estela 10 se realizó durante la mañana del 12 de agosto. La visita a la estela 12 se realizó durante la mañana del 13 de agosto.

En ambos casos (la línea de vista desde la Estela 12 hacia la Estela 10 y viceversa), la estela observada no se encuentra en el punto más alto de la montaña, sino que hay montaña atrás y arriba de ellas (Imagen 4), por lo que más allá de localizar el acimut de las estelas una respecto a la otra, habría que tratar de determinar la altura sobre el horizonte de la montaña posterior a la estela.

El procedimiento de análisis utilizó varias alturas para hacer las estimaciones, como se muestra más adelante, pero es recomendable en una futura visita llevar un instrumento que permita medir con precisión la diferencia de altura angular de una estela respecto a la otra.

Los datos de la situación geográfica de ambas estela son:

ESTELA 10 - Latitud: $14^{\circ} 50.576^{\prime} \mathrm{N} \quad$ Longitud: $89^{\circ} 11.066^{\prime} \mathrm{W}$

ESTELA 12 - Latitud: $\quad 14^{\circ} 50.029^{\prime} \mathrm{N} \quad$ Longitud: $89^{\circ} 07.449^{\prime} \mathrm{W}$

A partir de estos datos, se puede determinar el acimut relativo de las estelas, para así poder comparar la posición del sol en estos ángulos. 


\section{Estimación del acimut de cada una de las estelas (10 y 12) respecto a la otra}

Para determinar el acimut, a partir de las coordenadas geográficas, es necesario utilizar trigonometría esférica.

Sean el Polo Norte de la Tierra, la Estela 10 y la Estela 12 los tres vértices de un triángulo esférico, donde se utiliza el Polo Norte por ser uno de los polos del sistema de coordenadas utilizado (coordenadas geográficas). Sean:

$$
\begin{aligned}
& \text { L1 }=\text { distancia angular entre estelas } 10 \text { y } 12 \\
& \text { L2 }=\text { distancia angular Polo Norte - Estela } 10 \\
& \text { L3 = distancia angular Polo Norte - Estela } 12
\end{aligned}
$$

$A 1, A 2$ y $A 3$ son los ángulos opuestos a los lados $L 1$, L2 y $L 3$ respectivamente, donde los tres lados son segmentos de círculos mayores.

Es fácil obtener la amplitud de dos lados y un ángulo del triángulo, lo cual es suficiente para resolver el triángulo completo. Se deduce entonces que ${ }^{2}$ :

$$
\begin{aligned}
& L 2=90^{\circ}-f 10=90^{\circ}-14.8429^{\circ}=75.1571^{\circ} \\
& L 3=90^{\circ}-f 12=90^{\circ}-14.8338^{\circ}=75.1662^{\circ} \\
& A 1=I 10-I 12=89.1844^{\circ}-89.1242^{\circ}=0.0603^{\circ}
\end{aligned}
$$

Se muestra en la Imagen 5 el ángulo de interés, en color rojo, que es el acimut de la posición de la Estela 10 cuando el observador se encuentra en la Estela 12. Aplicando la ley de cosenos primero, y la ley de senos después, para triángulos esféricos, se obtiene lo siguiente:

$$
\begin{aligned}
& \operatorname{cosL} 1=\operatorname{cosL} 2 \operatorname{cosL} 3+\operatorname{sen} \mathrm{L} 2 \operatorname{senL} 3 \cos \mathrm{A} 1 \\
& \cos L 1=\cos \left(75.1571^{\circ}\right) \cos \left(75.1662^{\circ}\right)+\operatorname{sen}\left(75.1571^{\circ}\right) \operatorname{sen}\left(75.1662^{\circ}\right) \\
& \cos \left(0.0603^{\circ}\right)
\end{aligned}
$$

$L 1=0.05898^{\circ}=3^{\prime} 32.32 "$, que es el ángulo entre las Estelas 10 y 12 medidas desde el centro de la Tierra ${ }^{3}$.

\footnotetext{
2 Sea 010 la latitud de la Estela $10=14^{\circ} 50.576^{\prime}=14.8429^{\circ}$, 110 la longitud de la Estela $10=89^{\circ} 11.066^{\prime}=89.1844^{\circ}, 012$ la latitud de la Estela $12=14^{\circ} 50.029^{\prime}=14.8338^{\circ}$ y $₫ 12$ la longitud de la Estela $12=89^{\circ} 07.449^{\prime}=89.1242^{\circ}$

${ }^{3}$ Nótese que este resultado equivale a $0.05898^{\circ} 00.00103$ radianes, lo que multiplicado por el radio de la Tierra resulta en $6.57 \mathrm{Km}$, que es la distancia entre ambas estelas.
} 
$\operatorname{sen} A 2=\operatorname{sen} A 1 \operatorname{senL} 2 / \operatorname{sen} L 1=\operatorname{sen}\left(0.0603^{\circ}\right) \operatorname{sen}\left(75.1571^{\circ}\right) / \operatorname{sen}\left(0.05898^{\circ}\right)$

$$
A 2=81.1214^{\circ}
$$

Acimut $=360^{\circ}-\mathrm{A} 2=360^{\circ}-81.1214^{\circ}=278.8786^{\circ}$, que es el acimut de la Estela 10 encontrándose el observador en la Estela 12.

De la misma manera se puede estimar el acimut de la Estela 12 si el observador se encuentra en la Estela 10:

$$
\begin{gathered}
\operatorname{sen} A 3=\operatorname{sen} A 1 \operatorname{sen} L 3 / \operatorname{sen} L 1=\operatorname{sen}\left(0.0603^{\circ}\right) \operatorname{sen}\left(75.1662^{\circ}\right) / \operatorname{sen}\left(75.1571^{\circ}\right) \\
\text { Acimut }=A 3=81.1368^{\circ}
\end{gathered}
$$

Nótese que como todo está medido sobre la superficie de una esfera, el acimut medido desde una de las estelas no necesariamente debe ser igual a $360^{\circ}$ menos el acimut medido desde la otra estela.

\section{Estimación de las declinaciones de la esfera celeste en función de la altura}

La declinación de un cuerpo en la esfera celeste se puede calcular mediante la siguiente fórmula:

send =senf senh $+\operatorname{cosf} \cosh \cos A$, donde $f$ es la latitud del lugar de observación, $h$ la altura en grados sobre el horizonte del objeto astronómico y A es el acimut medido desde el norte en sentido horario (si A es medido desde el sur, como ocurre en ciertas convenciones, el $\cos \mathrm{A}$ de la expresión se convierte en - $\cos \mathrm{A}$ ).

Si se toma como punto de observación la Estela 12, se tienen los siguientes datos:

$$
f=14.8338^{\circ}
$$

$\mathrm{A}=278.8787^{\circ}$, medidos desde el $\mathrm{N}^{2}$

$\mathrm{h}=$ altura aparente del objeto sobre el horizonte

El cálculo de h puede volverse algo complejo si se incluye el efecto de la refracción atmosférica e incluso el tamaño del disco solar. Por ejemplo, un observador percibe la salida del sol antes que éste realmente esté saliendo por el horizonte verdadero. Esto ocurre porque la atmósfera hace ver levemente más arriba los cuerpos astronómicos que lo que realmente están. Por ejemplo la salida del sol se detecta aproximadamente en el momento en que el centro del disco solar se 
encuentra a 50 minutos de arco bajo el horizonte, 34 minutos por el efecto de la refracción y 16 minutos por ser el radio aparente del disco solar.

La refracción se puede aproximar por la fórmula de Bennet:

$$
R=\frac{60^{\prime \prime}}{\tan \left(h_{0}+\frac{7.31}{h_{0}+4.4}\right)}
$$

\section{Análisis de posición de las estelas 10 y 12 respecto a puntos de salida y puesta del Sol}

Apoyados en la fórmula de Bennett y en la trigonometría esférica se obtienen los resultados de las tablas 1, 2 y 3 :

$g$ - Representa la altura real del Sol sobre el horizonte. La prueba se hace con varias alturas pues se trabaja sobre la hipótesis de que los mayas utilizaban las posiciones de salida y puesta del Sol como referencia localizada a través de la montaña tras la cual salía y no como una proyección del punto del horizonte donde salía el Sol tras la montaña.

$R$ - Refracción, calculada en minutos de arco y estimada a través de la fórmula de Bennett. Indica cuántos minutos de arco eleva la atmósfera aparentemente la posición de un objeto en el espacio. Por ejemplo, si la posición real de una estrella es $4^{\circ}$ sobre el horizonte, la refracción hace que este cuerpo se vea en realidad $4^{\circ} 11.74^{\prime}$ sobre el horizonte.

+16' - En el caso del Sol, las coordenadas que se obtienen de programas y efe mérides es la posición del centro del disco solar. Al ser el Sol un disco de tamaño aparente de 32' de arco, entonces el centro del disco solar se encontrará $16^{\prime}$ de arco del punto del disco solar que se ve exactamente al momento de la salida de éste sobre el horizonte. Por ejemplo, cuando una persona ve salir el Sol, el centro de éste se encuentra aproximadamente 50 ' de arco bajo el horizonte, o sea los 34' de la refracción más los 16' del semidisco.

H centro Sol (') - Indica en minutos de arco la posición real del Sol cuando éste tiene la altura aparente en grados, indicada en la primera columna. El equivalente en grados y minutos se indica en la columna en amarillo con encabezado ${ }^{\circ}$. 
d - Pasando a coordenadas ecuatoriales, es la declinación de un cuerpo que se encuentra a la altura indicada en la columna amarilla y a un acimut de $278.88^{\circ}$ que es la posición de la Estela 10 respecto a la Estela 12. En la columna con encabezado ${ }^{\circ}$ " "aparece la conversión a grados, minutos y segundos.

Las columnas siguientes muestran los resultados a analizar. En las columnas cuyo encabezado es FECHA (aprox al 2010) se muestra la fecha de salida del Sol sobre la Estela 10 si se está observando desde la Estela 12, en función de la altura de la montaña tras la estela. Por ejemplo si la altura sobre el horizonte de la montaña tras la Estela 10 es de $6^{\circ}$, el Sol sale exactamente sobre ese punto, visto desde la Estela 12, las fechas 15 de abril y 27 de agosto.

Por supuesto, al tener la hipótesis que fue un marcador maya para las posiciones solares hay que determinar qué movimientos seculares de la Tierra pueden influir en mayor grado en los puntos de salida y puesta del Sol. Se tendrá en cuenta los movimientos de precesión y el cambio de inclinación del eje terrestre.

En el caso del Sol, la precesión no afecta los puntos de salida y puesta del Sol a través de los siglos. La precesión sólo afecta la posición del Sol y del punto Aries respecto al fondo estelar, pero el Sol siempre se movería en una franja delimitada por la inclinación del eje de la Tierra, o sea entre aproximadamente una declinación de $-23.5^{\circ}$ y $23.5^{\circ}$.

En cambio, la variación de la inclinación del eje terrestre sí hace variar los puntos de salida y puesta del Sol a través de los siglos. Para el caso, la inclinación actual del eje terrestre es de $23^{\circ} 26^{\prime}$. En el año 500 , dentro del período clásico maya, esta inclinación era de $23^{\circ} 38^{\prime}$. La diferencia es de $12^{\prime}$ de arco, apenas detectable por el ojo, pero esta quinta parte de grado de diferencia mueve las fechas estimadas en la tabla en aproximadamente dos días para el año 500 respecto a la actualidad.

En parte, esta combinación de movimientos puede resultar algo confusa, pero hay que examinar la dependencia de los movimientos aparentes del Sol respecto a diferentes variables. Los solsticios, tanto el de invierno como el de verano, se dan cuando el Sol tiene sus salidas y puestas extremas respecto a los puntos Este y Oeste. Nótese que la declinación del Sol durante los solsticios equivale a la inclinación del eje terrestre. Para la época actual, la declinación del Sol en el cielo corresponde $a+23^{\circ} 26^{\prime}$ durante el solsticio de verano, y a $-23^{\circ} 26^{\prime}$ durante el 
solsticio de invierno, dado que la inclinación del eje terrestre respecto a la normal de la eclíptica es precisamente de $23^{\circ} 26^{\prime}$ en la actualidad. $O$ sea que los puntos de salida y puesta del Sol son dependientes de la inclinación del eje terrestre.

Es independiente el trato que se puede dar a la precesión. Esto se puede determinar de manera más sencilla examinando la relación de la precesión respecto a la variación de las coordenadas absolutas ecuatoriales del Punto Aries, o sea la posición del Sol durante el equinoccio de primavera. Nótese que la precesión hace mover el Punto Aries, 50 segundos de arco al año por sobre la eclíptica, pero siempre resultando en la intersección entre la eclíptica y el ecuador celeste. ¿Qué quiere decir esto? Que el punto Aries siempre conserva su declinación igual a cero y esto confirma su independencia respecto a los puntos de salida y puesta del Sol. El punto Aries originalmente tenía como fondo la constelación de Aries, actualmente es la constelación de Piscis y en un futuro será la constelación de Acuario, con el paso de los siglos. Varía su posición sobre la eclíptica pero no varía su declinación. En conclusión, no afecta los puntos de salida y puesta del Sol.

Observando las fechas y tomando en cuenta la altura de la montaña tras la Estela 10, se concluye que no hay un evento astronómico tal como equinoccios, solsticios o pasos del Sol por el cenit que esté relacionado con la posición de estas dos estelas.

\section{Análisis de posición de las estelas 10 y 12 respecto a puntos de salida y puesta de diferentes estrellas: observaciones desde la Estela 12}

Para otras estrellas que no sean el Sol, el único cambio en el análisis es que al ser las estrellas un punto en el cielo y no un disco como en el caso del Sol, ya no hay que sumar los 16 ' de arco en el cálculo.

Si se trabaja bajo el supuesto que las estelas marcaban puntos de salida y puesta de estrellas brillantes, hay que relacionar la declinación en la salida con las estrellas brillantes del cielo. A las estrellas no les afecta en su posición la variación de la inclinación del eje de la Tierra pues esta inclinación es un ángulo entre la eclíptica y el ecuador celeste que sólo afecta al Sol. En cambio la precesión sí influye pues esta precesión "mueve" el fondo estelar al variar la posición de las referencias como por ejemplo el polo norte celeste (en el año 500 la estrella polar se encontraba a casi $10^{\circ}$ del polo norte celeste).

Las estrellas de primera o segunda magnitud que se ponían tras la Estela 10 en el año 500 eran Betelgeuse, Altair, Procyon y Aldebarán, aunque no en las posiciones exactas tras la estela sino a unos pocos grados de ella, por lo que no 
hay evidencia suficiente para afirmar que las estrellas sirvieron como referencia en la posición de las estelas.

\section{Observaciones desde la Estela 10}

Siguiendo el mismo procedimiento que el realizado con la Estela 12, se logra determinar que el acimut de la Estela 12 respecto a la Estela 10 es de $81.1368^{\circ}$, y las fechas de las posiciones del Sol y las estrellas referidas a las declinaciones correspondientes se muestran en la tabla 3, llegando a la misma conclusión de la insuficiencia de evidencia para afirmar que ya sea el Sol, ya sea las estrellas, sirvieron como referencia en la posición de las estelas.

\section{Triangulaciones respecto a la posición de las estelas 10 y 12}

El mapa muestra ángulos que parten desde las estelas 10 y 12, que apuntan hacia ángulos aproximados de salida y puesta del Sol durante los solsticios (los ángulos más abiertos), los equinoccios (líneas horizontales) y el ángulo de salida-puesta del Sol el día del paso del Sol por el cenit y su recíproco (los ángulos intermedios).

Tratando de buscar una relación geográfica respecto al Parque Arqueológico, aparentemente relación con solsticios o con el paso del Sol por el cenit parece casi nula en la línea de vista de una estela respecto a la otra.

El punto que sí parece tener sentido es la relación al establecimiento de los límites del Parque donde la Estela 10 marca aproximadamente el paralelo norte al cual limita, por lo que desde dicha estela, y viendo el momento de la salida del Sol durante los equinoccios, se traza una línea que limita al norte con el Parque Arqueológico.

De manera análoga la Estela 12 marca el límite hacia el sur del Parque, por lo que desde esta estela y viendo la puesta del Sol durante los equinoccios, se traza una línea imaginaria que colinda con el límite sur.

\section{Discusión de resultados}

La cantidad de datos disponibles acerca de las estelas y sus posibles alineamientos se han ido volviendo más precisos a medida que va mejorando la tecnología con la que se hacen las mediciones respecto a datos que incluso aún se conservan acerca de estudios realizados hace un siglo o más. 
Los datos tomados requieren un extenso análisis, pero en lo que concierne a las Estelas 10 y 12, parte central del estudio, se tienen los siguientes resultados:

La observación de la Estela 10 desde la Estela 12 no implica ninguna fecha asociada con los eventos de posición del Sol tales como equinoccios, solsticios 0 pasos del Sol por el cenit. De igual manera, si se observan estrellas brillantes que pudieron haber servido de referencia para la colocación de las estelas, las mismas no tienen un recorrido que directamente tenga que ver con la Estela 10. Se puede confirmar esto mediante los resultados obtenidos, en los que se tomó en cuenta los efectos de la refracción atmosférica, de la variación de la inclinación del eje terrestre para las posiciones del Sol y la precesión para las posiciones de las estrellas.

La observación de la Estela 12 desde la Estela 10 arroja resultados similares a los anteriores, sin eventos de Sol o de estrellas asociados.

La conclusión más importante indica que la Estela 10 marca el límite norte del Parque Arqueológico. Si se traza una línea hacia el punto de salida del Sol el día del equinoccio, la línea trazada limita aproximadamente al norte del parque. De manera análoga la Estela 12 marca el límite Sur del parque, tomando en cuenta que al trazar una línea desde ella hacia el punto de puesta del Sol durante los equinoccios, la misma pasa aproximadamente al sur del parque.

Una hipótesis que queda abierta es que los alineamientos de una estela respecto a la otra con las puestas del Sol el 12 de abril y el 1 de septiembre, según el arqueólogo Sylvanus Morley (Aveny 2005), si bien no tienen que ver con eventos solares concretos, pudieran ser un indicador climatológico como el inicio de la estación de las lluvias, indicando que era el tiempo de la quema y limpieza de los campos, previa a la siembra de la milpa, por poner un ejemplo. No se dispone de suficientes datos en este trabajo para hacer tal afirmación.

Varias mediciones han de ser tomadas para poder profundizar en los estudios, en particular mejorar las coordenadas geográficas existentes de cada punto, la altura sobre el nivel del mar, así como un perfil del horizonte desde varios lugares críticos, para establecer la variación de la altura respecto al acimut de cada punto del horizonte. También deberá incluirse en estudios posteriores, más estelas que se encuentran fuera del Parque Arqueológico. 


\section{Agradecimientos}

Agradecemos de manera especial y particular a Don Vito Véliz, ya que sin su apoyo y motivación no se hubiese podido culminar con éxito el trabajo, principalmente basados en su vasto conocimiento en Antropología e Historia, y en particular por todo el acceso que nos brindó durante la visita a Copán Ruinas.

También debemos agradecer a Fernando López quien, de manera muy cordial, nos trasladó a la poco accesible Estela 12 mientras compartía sus conocimientos acerca de la cultura maya y de Copán.

\section{Bibliografía}

- Aveny, A. F. OBSERVADORES DEL CIELO EN EL MÉXICO ANTIGUO. México: Fondo de Cultura Económica. 2005.

- Bennet G G. THE CALCULATION OF ASTRONOMICAL REFRACTION IN MARINE NAVIGATION. Journal of Navigation. Cambridge University Press. 1982.

- Berrocoso, M; Ramírez, M E; Enríquez-Salamanca, J M; Pérez-Peña, A. NOTAS Y APUNTES DE TRIGONOMETRÍA ESFÉRICA Y ASTRONOMÍA DE POSICIÓN. Servicio de Publicaciones Universidad de Cádiz. España. 2003.

- Fash Jr, W L; long, K Z. MAPA ARQUEOLÓGICO DEL VALLE DE COPÁN. PROYECTO ARQUEOLÓGICO COPÁN. Secretaría de Estado en el Despacho de Cultura y Turismo. Tegucigalpa, Honduras. 1983.

- Instituto Hondureño de Antropología e Historia. PLAN DE MANEJO ZONAARQUEOLÓGICA DE COPÁN. Tegucigalpa, Honduras. 2005.

- Morley, S. G. THE INSCRIPTIONS AT COPAN. Carnegie Institution of Washington. Publication 219. Washington, D.C. 1920.

- Pineda de Carías, M. C., Veliz, V., \& Agurcia, R. ACERCA DE LAS OBSERVACIONES DEL SOL REALIZADAS EN LA GRAN PLAZA DEL PARQUE ARQUEOLÓGICO DE COPÁN RUINAS, HONDURAS. VOL XXI. YAXKIN. 2002.

- Sharer, R. THE ANCIENT MAYA. Stanford University Press. 6a edición. 2006.

- Véliz, V. RUDIMENTOS DE ANTROPOLOGÍA. Litografía López S de RL. Tegucigalpa, Honduras. 1988. 


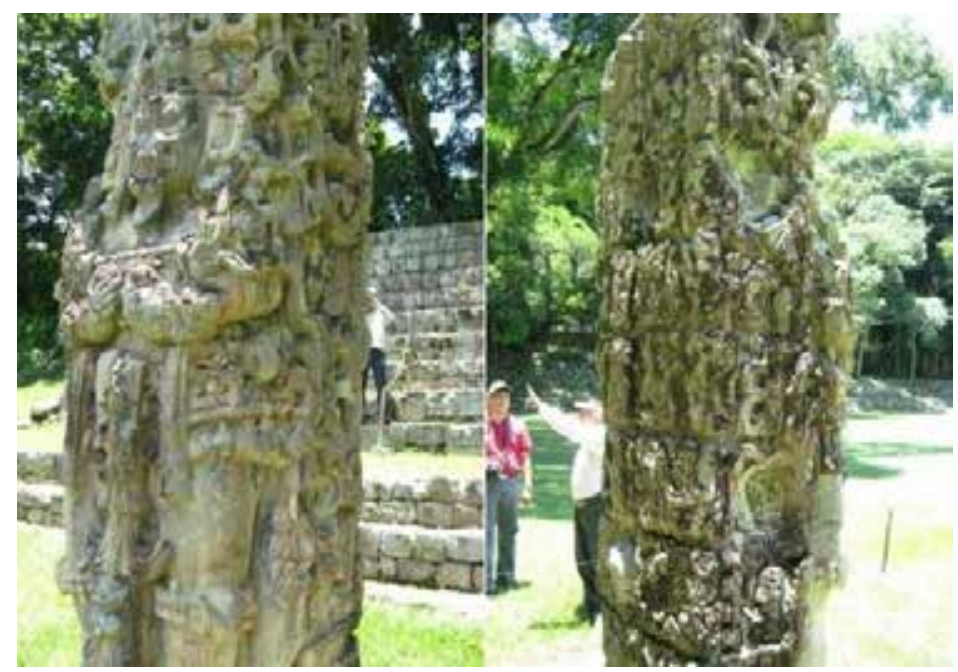

Imagen 1. Vista Sur-Este de la Estela $D$ (fotografía izquierda) y Norte-Oeste (fotografía derecha) a la hora del paso del Sol por el cenit. Fotografía: Roberto Schöngarth.

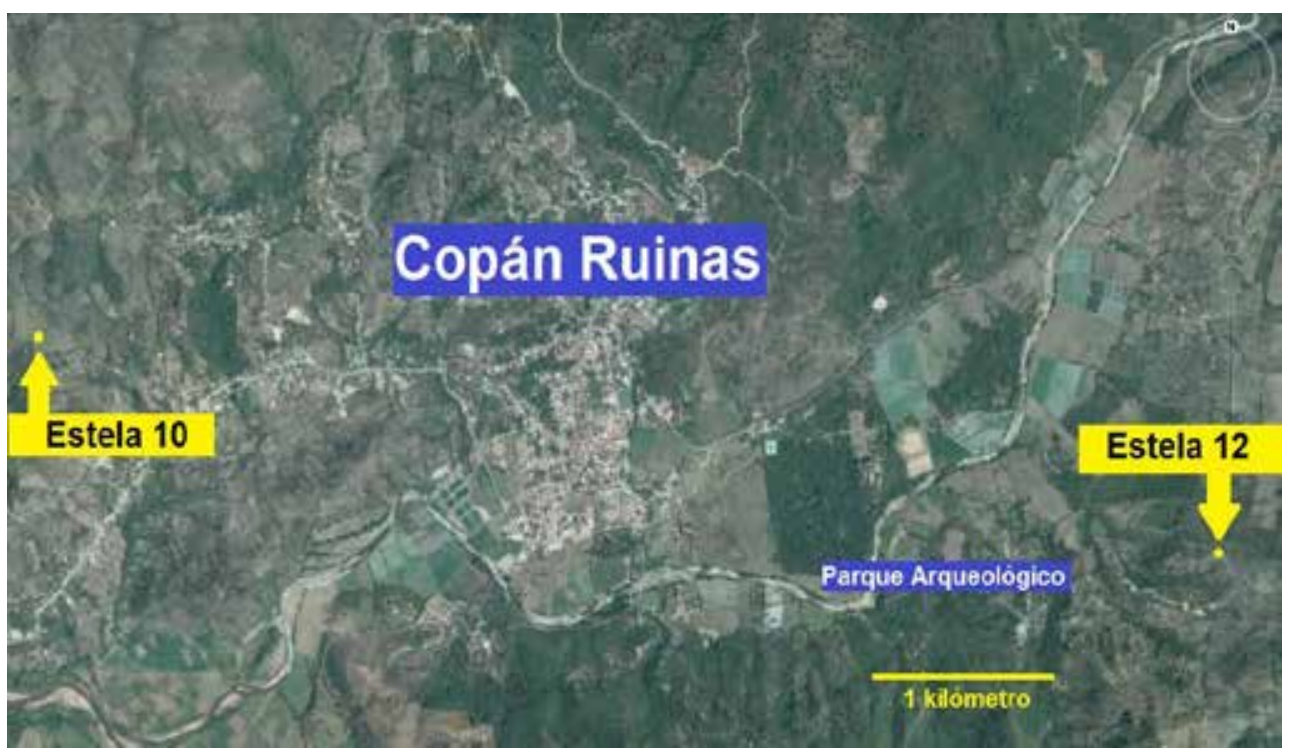

Imagen 2: Posición de Estelas D, 10 y 12 respecto al Parque Arqueológico y a Copán Ruinas. Fuente: Google Earth. 


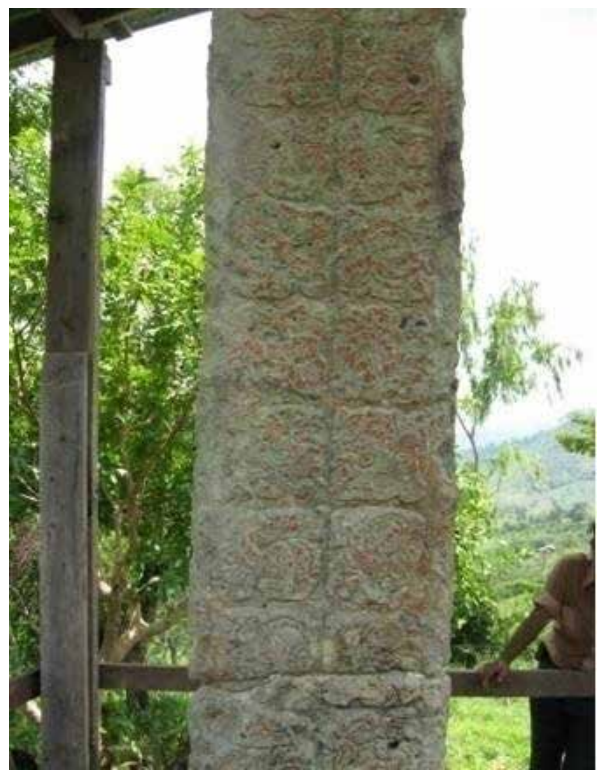

Imagen 3: Lado oeste de la Estela 12, "La Pintada". Fotografía: Roberto Schöngarth.

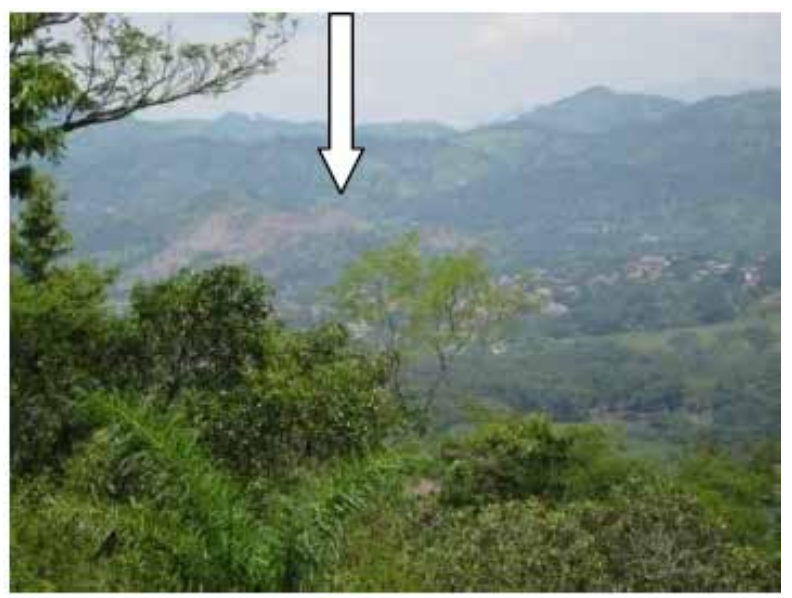

Imagen 4: Fotografía tomada desde la Estela 12 hacia la montaña de la Estela 10. La flecha indica la posición de la Estela 10, la cual no es visible a simple vista desde la Estela 12 debido a la vegetación. Fotografía: Roberto Schöngarth. 


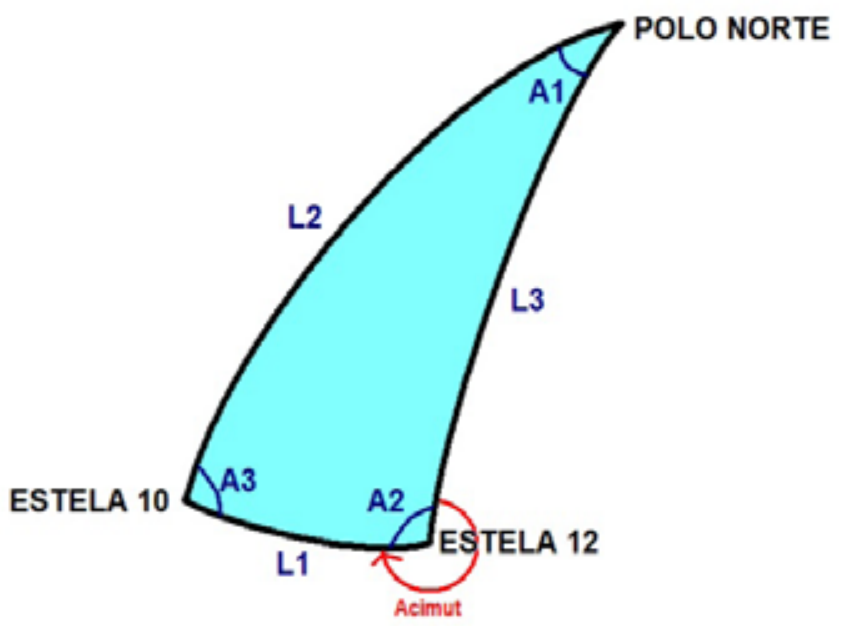

Imagen 5: Triángulo esférico para los cálculos de posición. Elaboración: Roberto Schöngarth.

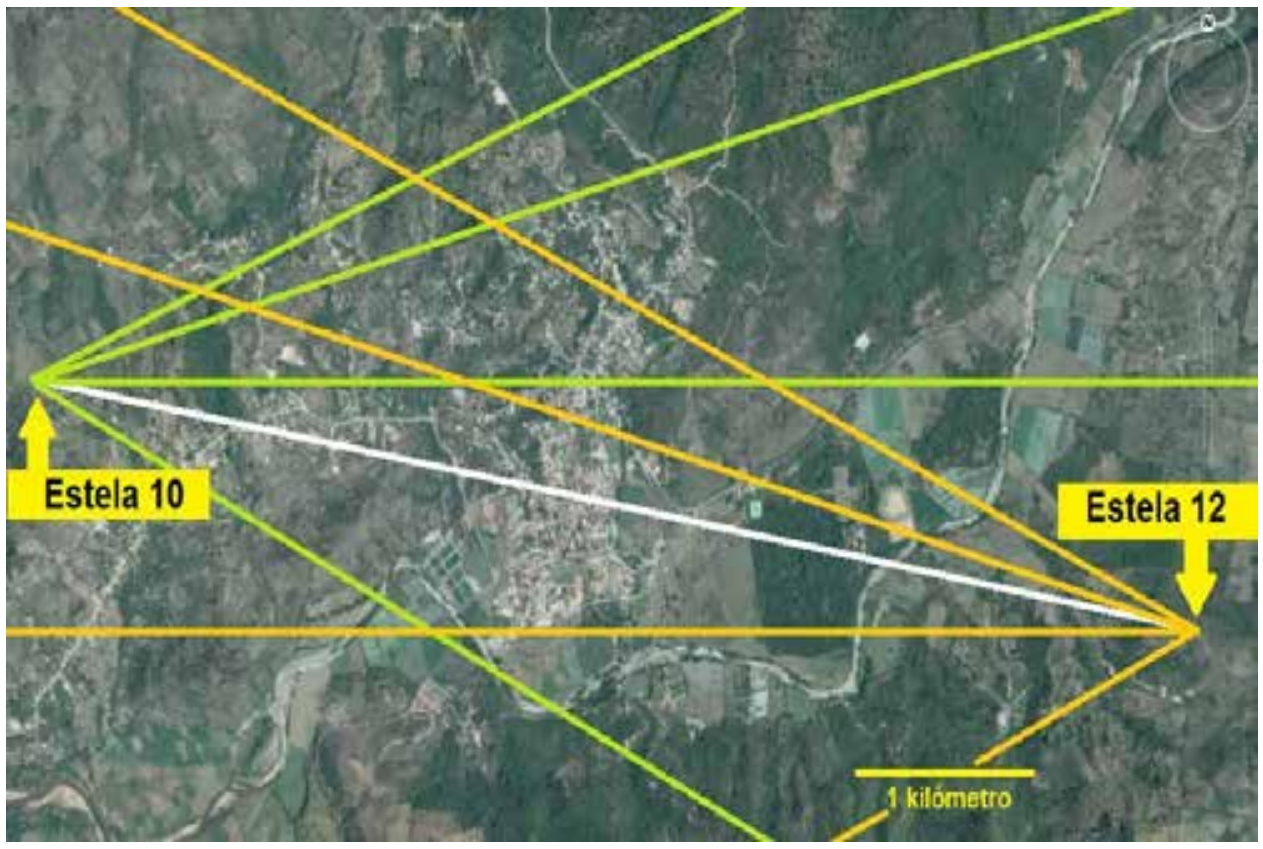

Imagen 6: Líneas de equinoccios, salidas y puestas de sol en su paso por el cenit y solsticios, trazadas desde estelas 10 y 12. Fuente: Google Earth. Cálculos: Roberto Schöngarth. 


\begin{tabular}{|c|c|c|c|c|c|c|c|c|c|c|c|c|}
\hline \multicolumn{13}{|c|}{ 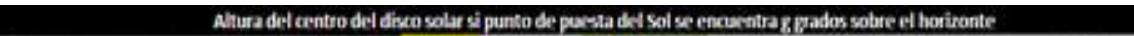 } \\
\hline हC) & Rlartmin) & $+16^{\prime}$ & hoentro 5017 ; & t & 8 & & ( & $\bar{l}$ & FECHA & $90 r 0 \times a 120 t 0)$ & FaCHA iapro & $2 x a|s 60|$ \\
\hline 0 & 4,48 & 30.48 & $.50,47753374$ & .50 .48 & 8.3617 & 8 & 21 & 42.30 & 11 de abril & 31 de agosto & 9 de abnil & 30 de anosto \\
\hline 1 & 24.33 & 40,33 & 3. 19.670577420 & o 12.67 & 8.6652 & 8 & 39 & 5467 & 12 de abril & 30 de agosto & 10 de abril & Y de aposto \\
\hline 2 & 18.22 & 34.22 & 85.75392371 & 1.78 & 8.945! & 8 & 56 & 5302 & 12 de abril & 30 de agosto & 11 de abril & 23 de aposto \\
\hline 3 & 14.34 & 30.34 & 148.65557762 & 229.56 & 22184 & 9 & 13 & 6.20 & 13 de abril & 29 de agosto & It de abril & 28 de agoste \\
\hline 4 & 12.78 & 27.74 & 24226397563 & 32.26 & 3.4005 & 9 & 28 & 4962 & 14 de abeil & 28 de igosto & 12 de abril & 27 de aposte \\
\hline 5 & 9.88 & 25.88 & 274.1168558 & 4.34 .12 & 2.7364 & 9 & 4 & 11.15 & 14 de abril & 27 de agosto & 11 de abril & 26 de aroste \\
\hline 6 & 8.51 & 2451 & 335.49110285 & 5. 35.49 & 9.9875 & 9 & 59 & 1691 & 1s de abril & 27 de agosto & 130 e abril & Xose aposto \\
\hline 7 & 7.45 & 23.45 & 39654526546 & 6. 3655 & 10.2342 & 10 & 14 & 216 & 16 de abcil & 26 de agosto & 14 de abril & zode agorto \\
\hline 8 & 6.62 & 22.62 & 457.37861527 & 73,38 & 10.4770 & 10 & 28 & $3) .19$ & 17 de abril & 25 de agosto & Is de abril & 24 de agosto \\
\hline 9 & 5.95 & 21.95 & $318 \cos 32643$ & 38.05 & 10.7160 & 10 & 42 & 57.70 & 17 de abtil & 25 de agosto & 16 de abril & 23 de angsto \\
\hline 10 & 5.39 & 21.39 & 578.60542452 & 938.61 & 109514 & 10 & 57 & 5.08 & 18 de abril & 24 de agosto & 16 de abril & 23 de agote \\
\hline
\end{tabular}

Tabla 1: Altura del centro del disco solar si punto de puesta se encuentra g grados sobre horizonte observado desde la Estela 12 hacia la 10. Elaboración: Roberto Schöngarth.

\begin{tabular}{|c|c|c|c|c|c|c|c|c|c|c|c|c|c|}
\hline $8(7)$ & S (arcemin) & $=0^{\circ}$ & h centro Sol 17 & + & + & 3 & $\cdot$ & ? & " & CSTRRLA la & al 2010) & CSTRELLA ২a & al 500 ) \\
\hline 0 & 34.48 & 34.48 & -34.47753374 & & -34.48 & 8.4313 & $\mathbf{8}$ & 25 & 52.51 & betelgeuse & $\cdot 7^{\prime} 24^{\prime}$ & Aetelgeuse & $-54^{\circ} 46$ \\
\hline 1 & 24.33 & 24,33 & 35.67087742 & 0 & 35.67 & 8.7339 & 8 & 44 & 2.13 & Altair & $+8^{+} 33^{\prime}$ & Altair & $+6^{2} 05$ \\
\hline 2 & 18.22 & 18.22 & 101.7839237 & 1 & 41,78 & 9.0161 & 9 & 0 & 57.80 & Regulus & $+11^{\prime} 58$ & Procyon & $+7^{4} 49$ \\
\hline 3 & 14.34 & 14.34 & 165.6555776 & 2 & 45.66 & 9.2856 & 9 & 17 & 8. 30 & & & Aldebaràn & $-12^{\prime} 14^{5}$ \\
\hline 4 & 22.74 & 12.74 & 228.2638756 & 3 & 48.26 & 9.5469 & 9 & $n$ & $\$ 2,01$ & & & & \\
\hline 5 & 9.88 & 9.88 & 290.1168558 & 4 & 50.12 & 9.0022 & 9 & is & 7.79 & & & & \\
\hline 6 & 8.51 & 8,51 & 351.4911028 & 5 & 51.49 & 10.0524 & 10 & 3 & 8.73 & & & & \\
\hline 7 & 7.45 & 7.45 & 412.5462654 & 6 & 52.55 & 10.2984 & 10 & 17 & 54,12 & & & & \\
\hline i & 6.62 & 6.62 & 473.3796152 & 7 & 53.34 & 20.5403 & 10 & 12 & 25.21 & & & & \\
\hline 9 & 5.95 & 5.95 & 534.0532643 & 4 & 54.05 & 20.7745 & 10 & ws & 42.72 & & & & \\
\hline 10 & 3.39 & 5.39 & 594.6084945 & 9 & 54.61 & 11.0131 & 11 & 0 & 47.02 & & & & \\
\hline
\end{tabular}

Tabla 2: Altura estrella si punto de puesta se encuentra g grados sobre el horizonte observando desde la estela 12 hacia la 10. Elaboración: Roberto Schöngarth. 


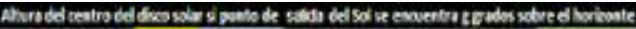

\begin{tabular}{|c|c|c|c|c|c|c|c|c|c|c|c|c|c|c|}
\hline & \multicolumn{2}{|r|}{$\cos$} \\
\hline 617 & Tibereiti & 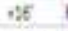 & houtosoln $=$ & & . & $h(n) d)$ & $x+5$ & 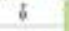 & * & * & Ffors is & to $x(20: 8)$ & Fors ineros & 580 \\
\hline 0 & 3628 & stas & so. 47755576 & -5008 & .08413 & 6.cts? & .01980 & $8 x+28$ & 848 & 8.10 .21 & 25 de letres & 25 te ocsove & is de'itrelo & Is de octible \\
\hline 1 & 2233 & 0.33 & 15.67cs772:0 & 1567 & 0.3273 & 0.0851 & $0: 278$ & \&spet & 830 & 6248 & 126 de lebrtis & 15 tc staget & 32 d 40020 & $124 \sec 2 \alpha$ \\
\hline$z$ & 20.22 & 3422 & 85765:3711 & 2538 & 2437 & 60250 & $0: 289$ & 82129 & 812 & 2.46 .35 & 27 te letros & it st wayst & is At 3tereic & 12 det schere \\
\hline$t$ & $\$ 634$ & 1034 & 10965557762 & 2964 & 24945 & 0,0435 & 0.3960 & -79521 & 75 & 59562 & 28 ft letres & Is te whave & 2t de letero & It te tetase \\
\hline 4 & 1174 & 2276 & 21216387563 & $12: 25$ & -3537 & 0.05:? & $0: 332$ & -7.6544 & 73 & $9.15,92$ & 28 dt febrite & 22 te ocsuyt & $27 d t^{2}+b+0$ & 10 ot sctabt \\
\hline 5 & 918 & $35: 83$ & $294: 6855 t 6$ & $16: 2$ & 45665 & $0.079 ?$ & 0.1284 & -7378 & $9 n$ & 21890 & I le rars & 12 se cesuare & 27 is lateres & 10 le sctade \\
\hline 6 & 85. & $245 !$ & $34529: 10285$ & 15,47 & 55315 & 0.9976 & -0.225 & $-2: 10: 0$ & .76 & 6266 & idt rarta & 11 te ectant & 28 st 5 teits & 9 it octert \\
\hline 7 & 2.85 & 13.45 & 536566.6546 & 1655 & 6509 & 0.1554 & 0.2188 & 68136 & 649 & 4. $35 \mathrm{ta}$ & I de rans & I0 thersay & Ithenass & Btiocsert \\
\hline 8 & 6.62 & 2262 & 652 396:5: 7 & 1734 & 7522 & ouse & $-0: 146$ & fsos! & +2 & 2425 & I At nert: & got octh't & 2ftrats: & 7 st sceart \\
\hline$\rightarrow$ & 535 & :1:s5 & 54405126438 & 1105 & 15342 & $0.500 t$ & $0.929 !$ & 6.1657 & 615 & 556.64 & 4 de marse & 9 de octubr & 2 stracso & 7 de ochert \\
\hline 20 & 330 & $2: 34$ & s7s 6cuteas 9 & 14:5: & 86435 & 0.68s & $.0: 013$ & $-53 t a 9$ & .550 & 9580 & . 5 st rans & A de octuen & 34 rass & 6 th sesert \\
\hline
\end{tabular}

\begin{tabular}{|c|c|c|c|c|c|c|c|c|c|c|c|c|c|c|}
\hline \multicolumn{13}{|c|}{ 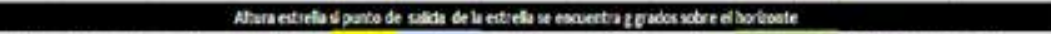 } & \multicolumn{2}{|c|}{ Etectoprecerios } \\
\hline t19 & Riart oil & $+\theta$ & hurebiln" & 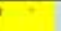 & ' & $\mathrm{h}$ ind & mb & 8 & 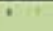 & 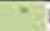 & ETR: & & Bसक & \\
\hline 0 & $3: 43$ & 32.3 & tadFss35\% & -34.48 & $0.57: 5$ & 0.0000 & $\cos 19$ & -87362 & 64 & 118 & Aipel & $411^{\prime}$ & 'Dipel & 1405 \\
\hline 1 & 2433 & 2433 & 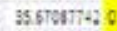 & 3567 & 0.5755 & D.8:D4 & $.0 .44 \%$ & 4.455 & 825 & 5269 & sita & 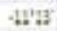 & (a) & $300^{\circ}$ \\
\hline 2 & 212 & un & - 102.7ss9sst 1 & $4: 7 t$ & 14954 & 00298 & $0: 418$ & $8: 421$ & 18 & 3408 & & & & \\
\hline 3 & 22.34 & 1434 & 1E5.6555776 2 & 22565 & 2.7508 & 00482 & $=01368$ & $7.86: 4$ & J 51 & 40.97 & & & & \\
\hline 4 & $12 \%$ & $: 274$ & 204:4847s 3 & 3425 & 2854 & 0.654 & 01390 & 75891 & 9.34 & $\operatorname{ses}$ & & & & \\
\hline 3 & $9 s 8$ & 2.81 & 2scisessst a & 5012 & . 3555 & 0.0sis & .01272 & $-2.305 t$ & 916 & 20.93 & & & & \\
\hline 5 & 15: & 35: & 55: 49:10:8 5 & 551,43 & 59592 & 0:1:21: & 02228 & 70095 & 71 & 4269 & & & & \\
\hline 7 & 7.65 & 2.25 & c:25et:65e 6 & $65: 55$ & 69735 & 0.1200 & .0217s & 6756 & $6+5$ & 215 & & & & \\
\hline 8 & 6.52 & $Q, 52$ & 473.5796:57 & 5355 & 73597 & 0.197 & 0.1127 & 6.17:t & $t 28$ & 18.3 & & & & \\
\hline 9 & 535 & 595 & 5320534663 & .5405 & 89003 & $0: 553$ & $0: 009$ & $619: 1$ & 611 & 3047 & & & & \\
\hline 20 & 589 & 559 & Sscoctigiss 9 & 9681 & (5.9.0: & 0.1790 & 0.0106 & 5.9205 & 5.54 & 39.87 & & & & \\
\hline
\end{tabular}

Tabla 3: Altura del centro del disco solar y de una estrella si punto de salida se encuentra g grados sobre el horizonte observado desde estela 10 hacia la 12. Elaboración: Roberto Schöngarth. 